\title{
Tratamento cirúrgico de ferimentos descolantes nos membros inferiores - proposta de protocolo de atendimento
}

\section{Degloving injuries of lower extremity - proposal of a treatment protocol}

\author{
Dimas André Milcheski ${ }^{1}$; Marcus Castro Ferreira, ECBC-SP²; Hugo Alberto Nakamoto 3 ; Paulo Tuma JR4; \\ ROLF GEMPERLI, TCBC-SP5
}

RE S U M O

\begin{abstract}
Objetivo: Apresentação e avaliação de protocolo para atendimento de pacientes com ferimentos descolantes dos membros inferiores através da retirada de enxerto de pele do tecido avulsionado e cobertura do leito cruento na fase aguda do trauma. Métodos: Este estudo avaliou retrospectivamente os pacientes com ferimentos descolantes em membros inferiores, em que o tratamento realizado baseou-se em protocolo de atendimento para ferimentos descolantes utilizado em nosso serviço,com pacientes atendidos na Unidade de Emergência. Resultados: Foram avaliados 21 pacientes. A etiologia do trauma foi atropelamento em 11 pacientes $(52,4 \%)$ e acidente de motocicleta em 10 (47,6\%). Os pacientes foram tratados conforme o protocolo apresentado a seguir: Os pacientes são inicialmente separados em instáveis e estáveis, conforme a condição hemodinâmica. Nos pacientes considerados instáveis (dois pacientes nesta casuística) realiza-se a retirada da pele e acondicionamento em banco de tecidos para enxertia posterior. Nospacientes considerados estáveis (19 pacientes) avalia-se a viabilidade do retalho através de parâmetros clínicos e do uso da fluoresceína. Se considerado viável, faz-se a sutura do retalho à posição original, e, se considerado inviável (todos os 19 pacientes), faz-se a ressecção e emagrecimento do tecido e realiza-se a enxertia da pele em malha com aplicação de curativo a vácuo sobre o enxerto. Conclusão: Os ferimentos descolantes devem ter seu atendimento padronizado a fim de se obter melhores resultados no tratamento e prevenir necrose da porção desenluvada e assim evitar nova área doadora para enxerto de pele.
\end{abstract}

Descritores: Ferimentos e lesões. Extremidade inferior. Transplante de pele. Cirurgia plástica.

\section{INTRODUÇÃO}

$\mathrm{O}$ $s$ ferimentos descolantes nos membros inferiores caracterizam-se frequentemente como lesões graves, e apresentam dificuldades de decisão sobre qual seria o tratamento mais adequado a ser instituído'.

Observamos, atualmente, um aumento da incidência de ferimentos descolantes. As lesões são secundárias à preensão da extremidade entre uma superfície móvel e uma superfície fixa como nos casos de atropelamentos. Há, frequentemente, alta energia envolvida, envolvimento de veículos pesados e alta velocidade associada à pouca proteção (motociclistas)². A pele e o subcutâneo desenluvados ficam presos apenas pela extremidade proximal ou distal do membro. Se não houver avaliação adequada e conduta correta, pode ocorrer insuficiência circulatória seja venosa ou arterial e evolução com necrose do tecido descolado 3 .

A circulação da porção avulsionada deve ser avaliada através de parâmetros clínicos, como o sangramento das bordas, a textura da pele e presença de trombose no plexo venoso subdérmico. Pode-se ainda utilizar a fluoresceína para corar a parte viável do tecido, permitindo assim a identificação da porção a ser ressecada do retalho 3 .

A limpezada ferida e o simples reposicionamento e sutura do retalho à posição original resultam geralmente na necrose do tecido avulsionado, levando à perda do retalho, maior risco de infecção, aumento da morbidade, necessidade de novas áreas doadoras, aumento do número de procedimentos cirúrgicos e prolongamento da internação. Segundo a literatura, a conduta mais apropriada consiste em completar a ressecção do tecido descolado, emagrecer este tecido, retirar a gordura deste tecido, deixando somente a pele com espessura total ou parcial e realizar a re-enxertia desta pele assim preparada como enxerto em malha, sobre o leito cruento, no mesmo ato cirúrgico ${ }^{1-5}$

Estas considerações são encontradas na literatura especializada de maneira esparsa, mas ainda não é usual

Trabalho realizado na Disciplina de Cirurgia Plástica da Faculdade de Medicina da Universidade de São Paulo, Hospital das Clínicas- SP-BR. 1. Médico Assistente da Disciplina de Cirurgia Plástica da Faculdade de Medicina da Universidade de São Paulo - FMUSP- SP-BR; 2. Professor Titular da Disciplina de Cirurgia Plástica da Faculdade de Medicina da Universidade de São Paulo - FMUSP- SP-BR; 3 . Médico Assistente da Disciplina de Cirurgia Plástica da Faculdade de Medicina da Universidade de São Paulo - FMUSP- SP-BR; 4. Médico Assistente da Disciplina de Cirurgia Plástica da Faculdade de Medicina da Universidade de São Paulo - FMUSP- SP-BR; 5. Professor Associado da Disciplina de Cirurgia Plástica da Faculdade de Medicina da Universidade de São Paulo - FMUSP- SP-BR. 
em nosso meio a aplicação destes princípios de tratamento, que têm sido utilizados, como protocolo, em nosso serviço desde 2003. Observamos em nosso serviço um número considerável de pacientes referidos tardiamente (mais de 24 horas) de outros centros médicos com ferimentos descolantes de membros inferiores que receberam o tratamento de limpeza local e reposicionamento e sutura do retalho. Nestes casos, houve um índice de necrose do retalho de $90 \%$, havendo necessidade de novas intervenções e criação de nova área doadora de pele.

Realizamos um estudo retrospectivo dos pacientes vítimas de ferimentos descolantes nos membros inferiores atendidos no Hospital das Clínicas da FM-USP no período compreendido entre julho de 2003 e junho de 2007 com análise do perfil epidemiológico e dos resultados do tratamento em pacientes em que nosso protocolo foi aplicado.

\section{MÉTODOS}

Foram estudados retrospectivamente prontuários referentes a 21 pacientes com ferimentos descolantes nos membros inferiores atendidos primariamente na Unidade de Emergência do Hospital das Clínicas da Faculdade de Medicina da USP durante o período de quatro anos, compreendido entre julho de 2003 e junho 2007 . O estudo está registrado na Comissão de Projetos de Pesquisa do HC-FMUSP sob o número0833/07.

O critério de inclusão adotado foi a presença do ferimento descolante em pelo menos um dos membros inferiores atendidos dentro das 24 horas iniciais do trauma. Foram excluídos os casos com presença de ferimento descolante em outra região anatômica, aqueles cujos registros não eram satisfatórios para os dados avaliados e os casos de ferimentos descolantes transferidos de outro serviço com mais de 24 horas de trauma.

Os dados referentes a aspectos epidemiológicos (idade, sexo e etiologia do trauma), tempo decorrido entre o trauma e a admissão hospitalar e a associação com outros traumas foram avaliados. Os dados referentes ao tratamento, a evolução, o tempo de internação e o seguimento destes pacientes também foram estudados.

\section{Protocolo de Atendimento}

O diagrama a seguir expõe o protocolo utilizado em nosso serviço para os pacientes com ferimentos descolantes de membro inferior (Figura 1).
O passo inicial do fluxo de atendimento distribui os pacientes em dois grupos: instáveis e estáveis. Os pacientes instáveis constituem aqueles com traumas graves e múltiplos, transfusões múltiplas, hipotermia e instabilidade hemodinâmica. O tratamento dos ferimentos descolantes neste grupo consiste na ressecção do tecido desenluvado e realização de curativo oclusivo na extremidade, liberando prontamente o paciente para a equipe da cirurgia do trauma realizar os procedimentos necessários à preservação da vida.

Em mesa auxiliar a equipe da cirurgia plástica realiza a limpeza e emagrecimento do retalho com a utilização de dermátomo elétrico, obtendo um enxerto de pele da porção descolada. Realiza-se então o acondicionamento do enxerto em um frasco com soro fisiológico e antibiótico (cefazolina e gentamicina) e coloca-se este frasco em geladeira no banco de tecidos para utilização posterior.

Assim que o paciente apresentar estabilidade e condições anestésicas (entre 24 e 72 horas), procede-se a enxertia da pele autógena conservada em banco de tecidos na forma de enxerto de pele espessura parcial em malha (trama de 1,5 a 3:1).

Os pacientes considerados estáveis tiveram inicialmente a avaliação da viabilidade do retalho avulsionado. Inicialmente observam-se fatores clínicos como a extensão do descolante, a qualidade do retalho (esmagado ou não), a posição do pedículo (anterógrado ou retrógrado), a espessura e largurada base do pedículo, o padrão de sangramento de bordas (arterial, venoso, isquêmico ou congesto) e o estado do plexo venoso subdérmico (trombose ou não). Pode se também utilizar a fluoresceína intravenosa $(1,5 \mathrm{mg} / \mathrm{kg})$ para avaliação da perfusão arterial do retalho avulsionado. Após 15 minutos observa-se se houve coloração do retalho total ou parcial através de lâmpada de fenda a fim de definir a parte viável do tecido e permitir a identificação da margem a ser ressecada do retalho.

Se considerado viável, o retalho é reposicionado ao seu leito e suturado, após os cuidados de limpeza com soro fisiológico sob pressão e desbridamento do leito da ferida e das bordas do retalho. Se considerado inviável, o retalho cutâneo é inicialmente lavado e é feito o desbridamento do leito da ferida e do retalho avulsionado com soro fisiológico sob pressão e em grande quantidade.O tecido ressecado é então submetido à retirada do enxerto de espessura de pele parcial com a utilização de dermátomo elétrico ainda quando o retalho está ligado à

Tabela 1 - Dados epidemiológicos referentes aos pacientes estudados.

\begin{tabular}{lcccc}
\hline Sexo $(\%)$ & Idade (média) & Etiologia (\%) & $\begin{array}{c}\text { Presença de traumas } \\
\text { associados (\%) }\end{array}$ & $\begin{array}{c}\text { Tempo médio entre } \\
\text { trauma e admissão } \\
\text { ao PS (horas) }\end{array}$ \\
\hline $\begin{array}{l}\text { Masculino }=13(61,9 \%) \\
\text { Feminino }=8(38,1 \%)\end{array}$ & $\begin{array}{c}32,9 \text { anos } \\
(4 \text { a } 73)\end{array}$ & $\begin{array}{c}\text { Atropelamento }=11(52,4 \%) \\
\text { Ac. motocicleta }=10(47,6 \%)\end{array}$ & 20 & 4,9 \\
\hline
\end{tabular}




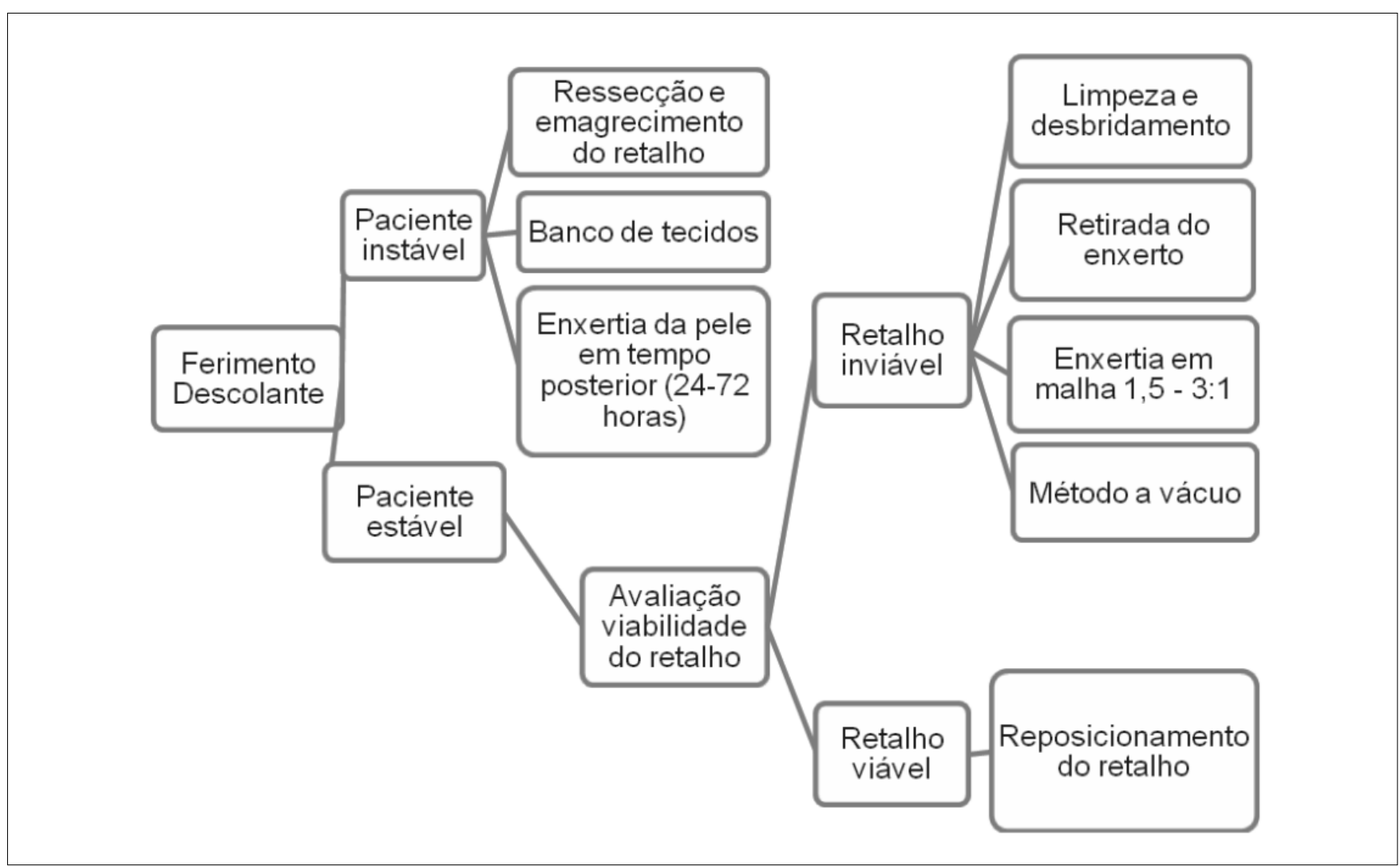

Figura 1 - Protocolo de atendimento - ferimentos descolantes nos membro inferior. Hospital das Clínicas - FMUSP.

extremidade. A pele é então preparada na forma de maIha 1,5 a 3:1 e enxertada ao leito da ferida. Realiza-se a fixação do enxerto às bordas da ferida com sutura para evitar o deslizamento do enxerto e aplica-se, de maneira rotineira, terapia com pressão negativa (aparelho a vácuo).

A abertura do curativo é realizada no quinto dia pós-operatório à beira do leito. Nos casos com perda parcial ou em que não houve pele suficiente para cobrir toda ferida, foram realizados curativo, desbridamento e nova enxertia com pele coletada de área doadora do paciente (geralmente coxa contralateral).

\section{RESULTADOS}

Os dados referentes aos aspectos epidemiológicos, ao tempo decorrido entre o trauma e a admissão hospitalar e às lesões associadas estão inseridos na tabela 1.

O tratamento oferecido no Hospital das Clínicas na fase aguda consistiu em limpeza e desbridamento do tecido inviável e realizadas no centro cirúrgico em todos os pacientes. Dentre os pacientes atendidos, dois foram considerados instáveis $(9,5 \%)$ e 19 estáveis $(90,5 \%)$. Com relação à viabilidade do retalho, todos os pacientes tiveram o retalho desenluvado considerado inviável após a avaliação intra-operatória do mesmo.
A tabela 2 ilustra o tratamento cirúrgico oferecido aos pacientes.

No atendimento inicial 18 pacientes foram submetidos ao protocolo com enxertia, três tiveram cobertura com retalhos devido à exposição de estruturas nobres e um caso apresentou combinação de enxerto e retalho.

A integração do enxerto de pele foi total em oito $(44,4 \%)$ casos e parcial em 10 pacientes (55,6\%), com perda estimada entre 5 e $10 \%$ do enxerto. Não houve perda completa do enxerto ou superior a $10 \%$ em nenhum paciente.

A tabela 3 demonstra as complicações, a taxa de amputação e a taxa de óbito observadas.

Dentre as complicações, 10 delas foram perdas parciais de enxerto. As amputações foram relacionadas ao trauma direto (três casos), à trombose arterial (um caso), à osteomielite (um caso) e à tetraplegia e exposição óssea (um caso).

Os óbitos foram secundários à hemorragia aguda, sepse e insuficiência renal aguda, com um caso cada.

A tabela 4 mostra o tempo médio de internação e de seguimento dos pacientes.

\section{DISCUSSÃO}

Os ferimentos descolantes ocorrem predominantemente em homens jovens (masculinos=61,9\%; idade 
Tabela 2 - Tratamento cirúrgico dos ferimentos descolantes.

\begin{tabular}{lccc}
\hline Enxertia & $\begin{array}{c}\text { Cobertura com } \\
\text { retalho }\end{array}$ & $\begin{array}{c}\text { Enxertia complementar } \\
(\mathrm{n}=18)\end{array}$ & $\begin{array}{c}\text { Area doadora } \\
\text { (primeira enxertia) }\end{array}$ \\
\hline $18(85,7 \%)$ & $4(19 \%)$ & $10(55,5 \%)$ & $\begin{array}{c}\text { Tecido desenluvado }=17(94,4 \%) \\
\text { Coxa }=1(5,6 \%)\end{array}$ \\
\hline
\end{tabular}

Tabela 3 - Complicações, amputações e taxa de óbito.

\begin{tabular}{ccc}
\hline Complicações & Amputações & Taxa de óbito \\
\hline 11 & 6 & 3 \\
$(52,4 \%)$ & $(28,6 \%)$ & $(14,3 \%)$ \\
\hline
\end{tabular}

média=32,5 anos), gerando um custo elevado para sociedade em relação a perda de dias trabalhados e aos custos relacionados ao tratamento ${ }^{1,2,4}$.

Os pacientes acometidos com ferimentos descolantes de membros inferiores apresentam frequentemente lesões associadas (95,2\%, nesta casuística) nas extremidades, geralmente fraturas, e sistêmicas, caracterizando traumatismos múltiplos ${ }^{3,4}$. A ocorrência de traumas associados dificulta o tratamento e aumenta o risco de amputação e óbito (28,6\% 14,3\%, respectivamente,nesta casuística). Faz-se importante o reconhecimento de condições associadas, bem como a implementação de condutas apropriadas através de protocolos de atendimento ao traumatizado e do envolvimento de equipe multidisciplinar (cirurgião do trauma, ortopedista, cirurgião vascular e cirurgião plástico).

As complicações encontradas na casuística apresentada foram nas sua maioria perda parcial de enxerto de pequena extensão (5 a 10\%; presentes em 10 pacientes)e passíveis de tratamento com curativo e nova enxertia (necessárias em 10 pacientes).

O tempo médio de internação (32,5 dias) foi elevado, mas não excessivo se o paciente for tratado corretamente com enxertia de pele no tempo adequado, e pode deixar o paciente suscetível a maior morbidade e complicações durante a internação Kudsk relatou em sua casuística tempo de internação de 68 dias² $^{2}$.

Devido ao aumento da incidência de ferimentos descolantes observada nos últimos anos e à falta de conduta uniforme oferecida a estes casos identificou-se a necessidade de padronização do atendimento. Dessa maneira, após a revisão da literatura e baseado na experiência do serviço, foi elaborado este protocolo de atendimento para os pacientes com ferimentos descolantes, discutido a seguir.

O passo inicial do fluxo de atendimento divide os pacientes em dois grupos, instáveis e estáveis. Os pacientes instáveis consistem nos casos de traumas graves e múltiplos, transfusões múltiplas, hipotermia e instabilidade hemodinâmica (assim considerados em conjunto pela equipe de cirurgia do trauma e da anestesia). Na casuística apresentada, dois casos se enquadram neste subgrupo.

O tratamento dos ferimentos descolantes neste grupo consiste na ressecção do tecido desenluvado e realização de curativo oclusivo na extremidade, liberando prontamente o paciente para a equipe da cirurgia do trauma realizar os procedimentos necessários à preservação da vida. Quando o paciente apresentar estabilidade (24 a 72 horas) realiza-se a enxertia de pele autógena conservada em banco de tecidos na forma de enxerto de pele espessura parcial em malha (1,5 a 3:1).A enxertia da pele autógena deve ser realizada o mais precoce possível, uma vez que há diminuição gradual da integração do enxerto com o passar dos dias. Com 14 dias o enxerto é considerado inviável e deve ser desprezado ${ }^{6-8}$.

O grupo de pacientes considerados estáveis deve ter inicialmente a avaliação do retalho avulsionado. Várias táticas são descritas para avaliação do retalho ${ }^{1-4,9}$. A utilização do corante fluoresceína não garante a viabilidade do retalho, uma vez que pode haver comprometimento da porção venosa, podendo ocorrer necrose devido à congestão ou trombose. Dessa maneira, há necessidade de se utilizar vários parâmetros e senso crítico para decidir entre o reposicionamento e sutura do retalho ou sua ressecção. O reposicionamento e sutura oferece melhor resultado funcional e estético, mas raramente é bem sucedido. Usualmente a decisão penderá para ressecção do retalho avulsionado, conduta esta adotada nos 19 pacientes estáveis atendidos.

Após a ressecção e emagrecimento do retalho, procede-se à utilização de terapia com pressão negativa a fim de aumentar a integração do enxerto. O aparelho a vácuo consiste em um método auxiliar que vem tendo

Tabela 4 - Tempo médio de internação e de seguimento.

\begin{tabular}{lcc}
\hline & Tempo médio de internação (dias) & Tempo médio de seguimento (meses) \\
\hline Médias & 32,5 & 6 \\
Variação & 2 a 62 & 1 a 26 \\
\hline
\end{tabular}


ampla aceitação em feridas difíceis, extensas e com leitos irregulares ${ }^{10}$. Em nosso meio foi primeiro utilizado por Ferreira et al. ${ }^{11,12}$. A terapia com pressão negativa proporciona imobilização do enxerto e permite a aspiração de debris, resultando em menor incidência de seroma e hematoma e em uma maior integração do enxerto. Também apresenta o efeito de diminuir a população bacteriana na ferida $^{13}$. Há evidência clínica e experimental suficiente na literatura médica para preconizar a utilização desse curativo $^{11,12,14-16}$. No estudo em questão o aparelho a vácuo foi utilizado de maneira rotineira nos casos atendidos.

Com a utilização deste protocolo obteve-se integração adequada da pele avulsionada e cobertura parcial ou completa do defeito já na fase inicial do atendimento proporcionando assim a utilização de pouca ou nenhuma extensão da uma área doadora de auto-enxerto.

\title{
A B S S T R A C T
}

\begin{abstract}
Objective: Degloving injuries on the lowerextremities are often serious injuries. It is difficult to decide on the most appropriate treatment, whether flap repositioning and suturing or converting the avulsed flap tosplit-thickness skingrafting. Methods: This study assessed patients with degloving injuries in lower extremities, reviewing the epidemiological profile and treatment performed. It is proposed a treatment protocol for management of those lower extremity avulsion injuries. Results: Twenty-one patients were evaluated. The cause of trauma was running over in 11 patients (52,4\%) and motorcycle accident in 10 (47,6\%). All twenty-one patients had treatment with washing, debridement, resection of avulsed flap and converting the flap to split-thickness graft, in according with the following treatment protocol for management of those lower extremity avulsion injuries that came to our Emergency Unit: Patients were initially classified as unstable or stable (hemodynamically). In the unstable group (two patients), due to the patient condition, flap resection was performed and the skin kept in the tissue bank for later grafting. In the stable group (19 patients), flap viability was assessed using clinical parameters and fluorescein. If deemed viable the flap was sutured to its original position. If deemed unviable (all 19 patients), it was resected and converted to split-thickness skin and mesh grafting with vacuumassisted device over the graft. Conclusion: In order to avoid flap necrosis and to add a new skin donor area is important to recognize the problem in the Emergency Room and to manage properly those injuries.
\end{abstract}

Key words: Wounds and injuries. Lower extremity. Skin transplantation. Surgery, plastic.

\section{REFERÊNCIAS}

1. Mandel M. The management of lower extremity degloving injuries. Ann Plast Surg. 1981; 6(1): 1-5.

2. Kudsk KA, Sheldon GF, Walton RL. Degloving injuries of the extremities and torso. J Trauma. 1981; 21(10): 835-9.

3. Widgerow $A D$, Chait LA. Degloving injuries and flap viability assessment. S Afr Med J. 1993; 83(2): 97-9.

4. Waikakul S. Revascularization of degloving injuries of the limbs. Injury. 1997; 28(4): 271-4

5. Huemer GM, Schoeller T, Dunst KM, Rainer C. Management of a traumatically avulsed skin-flap on the dorsum of the foot. Arch Orthop Trauma Surg. 2004; 124(8): 559-62. Epub 2004 Aug 3.

6. Cram $A E$, Domayer MA. Short-term preservation of human autografts. J Trauma. 1983; 23 (10): 872-3.

7. Sheridan R, Mahe J, Walters P. Autologous skin banking. Burns. 1997: 24(1): 46-8.

8. Nogueira A, Martínez MJ, Arriaga MJ, Pérez A. Delayed fullthickness autografting of cryopreserved avulsed skin in degloving injuries of the extremities. Plast Reconstr Surg. 2001; 107(4): 100913.

9. Letts RM. Degloving injuries in children. J Pediatr Orthop. 1986; 6(2): 193-7.

10. Ferreira MC, Tuma Jr P, Carvalho VF, Kamamoto F. Complex wounds. Clinics. 2006; 61(6): 571-8.

11. Ferreira MC, Wada A, Tuma Jr P. The vacuum assisted closure of complex wounds: report of 3 cases. Rev Hosp Clin. 2003; 58(4): 227-30.

12. Wada A, Ferreira MC, Tuma Jr P, Arrunátegui G. Experience with local negative pressure (vacuum method) in the treatment of complex wounds. São Paulo Med J. 2006; 124 (3): 150-3.
13. DeFranzo AJ, Argenta LC, Marks MW, Molnar JA, David LR, Webb $\mathrm{LX}$ et al. The use of vacuum-assisted closure therapy for the treatment of lower-extremity wounds with exposed bone. Plast Reconstr Surg. 2001; 108(5): 1184-91.

14. Josty IC, Ramaswamy, Laing JH. Vacuum-assisted closure: an alternative strategy in the management of degloving injuries of the foot. Br J Plast Surg. 2001; 54(4): 363-5.

15. DeFranzo AJ, Marks MW, Argenta LC, Genecov DG. Vacuumassisted closure for the treatment of degloving injuries. Plast Reconstr Surg. 1999; 104(7): 2145-8.

16. Scherer LA, Shiver S, Chang M, Meredith JW, Owings JT. The vacuum assisted closure device: a method of securing skin grafts and improving graft survival. Arch Surg. 2002; 137(8): 930-3; discussion 933-4.

Recebido em 03/04/2009

Aceito para publicação em 04/06/2009

Conflito de interesse: nenhum

Fonte de financiamento: nenhuma

\section{Como citar este artigo:}

Milcheski DA, Ferreira MC, Nakamoto HA, Gemperli PTR. Tratamento cirúrgico de ferimentos descolantes nos membros inferiores - proposta de protocolo de atendimento. Rev Col Bras Cir. [periódico na Internet] 2010; 37(3). Disponível em URL: http://www.scielo.br/rcbc

\section{Endereço para correspondência:}

Dimas André Mllcheski

E-mail: drdimasandre@gmail.com 